\title{
How many molecules are required to obtain a steady faradaic current from mediated electron transfer at a single nanoparticle on a supporting surface?
}

\author{
Enno Kätelhön * Kay J. Krause ${ }^{\dagger} \quad$ Bernhard Wolfrum ${ }^{\dagger \ddagger}$ \\ Richard G. Compton *§
}

\section{Abstract}

We investigate the chronoamperometric noise characteristics of electron transfer reactions occuring on single nanoparticles and assemblies of well-separated nanoparticles on a supporting surface. To this end, we combine a formerly described expression for the steady-state current of a single particle with the shot noise model and derive an expression for the signal-to-noise ratio as a function of bulk concentration and the particle's radius. Our findings are supported by random walk simulations, which closely match the analytical result.

\section{Introduction}

Due to its relevance to fundamental nanoelectrochemistry as well as to a variety of sensing applications, particle impact voltammetry has received growing attention during recent years. Studies hereby typically focus on faradaic currents that occur during collisions of nanoparticles (NPs) with the electrode, while currents may either result from the electrochemical interaction of the particle surface with species dissolved in the surrounding solution $[1,2,3,4,5,6,7]$ or from the nanoparticles' intrinsic electrochemistry [8,9].

This research was accompanied by a variety of theoretical studies supporting experimental research. Work on this topic includes investigations on steady state limiting currents of individual nanoparticles [10,11], voltammetry of arrays of

\footnotetext{
${ }^{*}$ Department of Chemistry, Physical and Theoretical Chemistry Laboratory, Oxford University, South Parks Road, Oxford OX1 3QZ, United Kingdom

${ }^{\dagger}$ Institute of Bioelectronics (PGI-8/ICS-8) and JARA-Fundamentals of Future Information Technology, Forschungszentrum Jülich, 52425 Jülich, Germany

${ }^{\ddagger}$ IV. Institute of Physics, RWTH Aachen University, 52074 Aachen, Germany

$\S$ corresponding author
} 
distributed nanoparticles [12, 13], steady state voltammetry [14], and studies on the electrode kinetics of individual nanoparticles [15].

Even though a variety of studies on particle impact voltammetry have been published, the faradaic current's noise characteristics have yet not been investigated. In this work, we focus on the typical experimental setup of a single nanoparticle or a set of diffusionally isolated nanoparticles on a supporting electrode surface, which for example is a good approximation for the reduction of hydrogen peroxide at AgNPs [6] or proton reduction at AuNPs [5]. We derive an expression for the signal-to-noise ratio and support our findings through random walk simulations.

\section{Theory}

\subsection{Theoretical Model}

We consider an irreversible one-electron process exclusively taking place on the surface of nanospheres supported on an electrode,

$$
A+e^{-} \rightarrow B
$$

where the rate of reaction is assumed to be infinite, i.e. molecules react instantaneously when they come in contact with a nanosphere and the reaction is fully diffusion controlled. The reaction is assumed not to occur on the supporting electrode at the potentials of intent. Furthermore, we consider a high electrolyte concentration that effectively screens all electric fields in solution. Thus, the analyte's mass transport to the nanoparticles is not facilitated by the electrode's electric field but limited to diffusive processes only.

Based on this model, we investigate the geometries of a single or multiple spherical nanoparticles $i$ that feature the radius $r_{i}$ and are attached to a supporting surface. In the latter case, nanoparticles are well-separated and their diffusion fields do not overlap during the experiment,

$$
d_{i j}+r_{i}+r_{j} \gg \sqrt{2 D t_{\max }}
$$

where $d_{i j}$ represents the distance between the nanoparticles $i$ and $j, D$ the analyte's diffuison coefficient, and $t_{\max }$ the duration of the experiment. An illustration of the investigated geometry can be found in Figure 1.

\subsection{Noise analysis}

The steady state current of the above mentioned model has formerly been investigated by two groups. In 1987 Bobbert et al. published an analytically found expression for the limiting current [10], which was later confirmed through a numerical approach by Streeter and Compton in 2007 [11]. In the following, we

base our analysis on this result. Accordingly, the steady state current $I_{l i m}$ of a single spherical electrode $i$ on a supporting surface is given by 


$$
I_{l i m}=4 \pi \ln (2) F D c^{*} r_{i}
$$

for the case of one electron being transferred [10], where $F$ represents the Faraday constant and $c^{*}$ the analyte's bulk concentration. Since the above mentioned condition for $d_{i j}$ in Formula (2) limits our analysis to spheres that are diffusionally isolated and hence do not interact with each other, we can superpose all Faradaic responses that occur at the considered $n$ spheres. The current caused by an assembly of $n$ well-separated spheres is then given by

$$
I_{l i m}=4 \pi \ln (2) F D c^{*} \sum_{n} r_{i}
$$

The system's noise characteristics can be described through the shot-noise model [16], which was introduced by Walter Schottky in 1918 and today is a commonly used noise model for a variety of different experimental settings. It generally applies to currents that are formed through discrete numbers of equal charge carriers that cross a potential barrier. The power spectral density $S_{n}$ of the current noise is then given by

$$
S_{n}=2 e_{0} I_{l i m}
$$

where $e_{0}$ represents the elementary charge [17]. We can now calculate the current's variance $\operatorname{Var}\left(I_{\text {lim }}\right)$ by integration of the power spectral density in the frequency regime

$$
\operatorname{Var}\left(I_{\text {lim }}\right)=\int_{f_{\min }}^{f_{\max }} d f S_{n}=2 e_{0} I_{l i m} \Delta f
$$

where $f_{\min }$ and $f_{\max }$ are the minimal and maximal frequencies that can be resolved by the respective measurement setup. $\Delta f$ represents the measurement's bandwidth and can be found as the difference between $f_{\min }$ and $f_{\max }, \Delta f=$ $f_{\text {max }}-f_{\text {min }}$.

We calculate the system's signal-to-noise ratio snr as the ratio between the average current $I_{\text {lim }}$ (see Formula (4)) and the current's standard deviation, which is given by square root of the expression derived in Formula (6). It then calculates to

$$
s n r=\frac{I_{\text {lim }}}{\sqrt{\operatorname{Var}\left(I_{\text {lim }}\right)}}=\sqrt{\frac{2 \pi \ln (2) F D c^{*} \sum_{n} r_{i}}{e_{0} \Delta f}}
$$

Hence, at a given set of particle radii $r_{i}$, the signal-to-noise ratio only depends on the analyte concentration $c^{*}$ and is proportional to its square root.

This expression already answers the question posed in the headline via an analytical model. Since the Faradaic current at an electrode always results from a discrete number of charge transfer events within in a given sampling interval, the detected current is therefore generally affected by random fluctuations and will never fully reach a noise-free state. However, the magnitude of the noise's 
power drops with increasing concentration and particle radii, and can be calculated through Formula (7). By this means, the number of molecules that are required to reach a certain noise level can be found for any given experimental conditions.

\subsection{Computational Methods}

In this work, we employ a simulation framework that has been previously discussed by Kätelhön et al. in 2013 [18]. The cited publication provides all simulation details and extensively discusses its applicability in nanoelectrochemistry and the modelling of noise characteristics. The authors further disclose the simulation's source code, which is available on request. Hence, we here limit our explanation to the most relevant characteristics of the simulation framework. In short, the Brownian motion of each analyte molecule is modelled individually for the time interval of the experiment. Hereby, the molecules three-dimensional trajectories are calculated through three one-dimensional random walks that each fulfil the following expression that can be derived from the general solution of diffusion equation

$$
d x_{1 D}=\sqrt{2 D d t}
$$

where $d x_{1 D}$ represents the one-dimensional spatial step width of the random walk and $d t$ the random walk's temporal step width. Molecules are further reflected at boundaries and electrodes, and react immediately and fully upon contact with an appropriately-biased electrode. The simulation code is written in $\mathrm{C}++$ while all data analysis is carried out in Matlab.

Figure 2 illustrates the simulated geometry in its initial state. The spherical electrode is enclosed in a cubic box that features the side length $A_{\max }$, while $A_{\max }$ is chosen to be large relative to the electrode's diffusion field:

$$
\frac{A_{\max }}{2}-r_{i}>6 \sqrt{D t_{\max }}
$$

All simulations use the diffusion coefficient of protons $\left(\mathrm{H}^{+}\right)$in water, $D=$ $9.3 \times 10^{-9} \mathrm{~m}^{2} \mathrm{~s}^{-1}[19]$, a side length $A_{\max }$ of $2000 \mathrm{~nm}$, model the first $2.7 \times 10^{-6}$ $\mathrm{s}$ of a chronoamperometric experiment, and use a one-dimensional step width 1 $\mathrm{nm}$. The noise analysis is based on the current in the time interval between $\mathrm{t}=$ $1.35 \times 10^{-6} \mathrm{~s}$ and $\mathrm{t}=2.7 \times 10^{-6} \mathrm{~s}$, hence, analysed currents are reasonably close to the steady state as discussed below. Furthermore, for reasons of computational efficiency, data sets of different analyte concentrations were obtained from the same simulations using varied numbers of molecule trajectories in the analysis.

\section{Results and Discussion}

We compare the analytical result of the signal-to-noise ratio to our random walk simulations of a diffusionally isolated nanoparticle on a surface. Hereby, we investigate spherical electrodes of radius $r_{i}=10 \mathrm{~nm}$ and $r_{i}=20 \mathrm{~nm}$ at 
different bulk concentrations $c^{*}$ of the analyte. In the simulated currents, we find that the chronoamperometric current response quickly approaches a steady state (see Supporting Material). Hence, we base the following noise analysis on the time interval between $\mathrm{t}=1.35 \times 10^{-6} \mathrm{~s}$ and $\mathrm{t}=2.7 \times 10^{-6} \mathrm{~s}$ to ensure that the system is close to the steady state. Figure 3 presents the so-obtained power spectral density of the current noise, where we find the expected white noise spectrum that was discussed above in Equation (5). Furthermore, the average power spectral density of the simulated noise trace closely matches the theoretical result.

A comparison between the resulting signal-to-noise ratios and the analytically obtained value is shown in Figure 4. Here, we again find a good agreement between the two graphs above a concentration of $0.4 \mathrm{mM}$. Below that concentration, the number of events within the simulated time interval is so small that there are not enough events to allow a proper statistical analysis (data not shown). However, the simulated signal-to-noise ratio for a radius of $10 \mathrm{~nm}$ is slightly underestimated, which is a results of the random walk model that generally underestimates faradaic currents with an increase in the ratio between the random walk's step width and the size of the three-dimensional electrode geometry under consideration. Since both simulations utilize the same step width of $1 \mathrm{~nm}$, this effect has a stronger impact on the current of the smaller sphere. Furthermore, we can find that the simulated graphs exhibits waves and individual data points are not statistically independent. This is due to the formerly mentioned method of data acquisition in the simulation: for reasons of computational efficiency, varied concentrations are obtained from the same simulation by using only a certain number of modelled trajectories. This results in a stochastic dependency of the individual points of the signal-to-noise ratio on each other, however, it does not impact the accuracy of each single point's value.

\section{Conclusions}

In this work, we investigate the noise characteristics of a single- and an assembly of well-separated spherical electrodes on a supporting surface. We derive an expression for the signal-to-noise ratio of the system's steady state current and compare our result to random walk simulations. Here, we find a good agreement between theory and simulation.

In conclusion, we show that number of molecules that are required to obtain a steady current can be calculated through the signal to noise ratio snr

$$
s n r=\sqrt{\frac{2 \pi \ln (2) F D c^{*} \sum_{n} r_{i}}{e_{0} \Delta f}}
$$

which is proportional to the square root of the analyte concentration $c^{*}$ and only depends on its value at given particle radii $r_{i}$ and given experimental conditions for $D$ and $\Delta f$. Since the Faradaic current is directly affected by the stochastic 
Brownian motion of the analyte molecules, it can never reach a fully noise-free state. However, the number of molecules that are required to reach a certain noise level can be calculated through the derived expression.

\section{Acknowledgments}

The research leading to these results has received partial funding from the European Research Council under the European Union's Seventh Framework Programme (FP/2007-2013) / ERC Grand Agreement n. [320403]. Kay J. Krause and Bernhard Wolfrum gratefully acknowledge funding by the Helmholtz Young Investigator program. We thank Mr Tom Bartlett for proofreading the manuscript.

\section{Keywords}

Nanoelectrochemisrty, nanoparticles, noise spectroscopy, random walks

\section{TOC}

Noise spectroscopy: We investigate the chronoamperometric noise characteristics of elektron transfer reactions occuring on single nanoparticles and assemblies of diffusionally isolated nanoparticles on a supporting surface and derive an expression for the current's signal-to-noise ratio.

\section{References}

[1] Michael Heyrovský, Jaroslav Jirkovský, and Miroslava Štruplová Bartáčková. Polarography and voltammetry of aqueous colloidal ti02 solutions. Langmuir, 11:4300, 1995.

[2] Michael Heyrovský, Jaroslav Jirkovský, and Bernd R. Müller. Polarography and voltammetry of aqueous colloidal sno2 solutions. Langmuir, 11:4293, 1995.

[3] Xiaoyin Xiao and Allen J Bard. Observing single nanoparticle collisions at an ultramicroelectrode by electrocatalytic amplification. Journal of the American Chemical Society, 129(31):9610-9612, 2007.

[4] Allen J Bard, Hongjun Zhou, and Seong Jung Kwon. Electrochemistry of single nanoparticles via electrocatalytic amplification. Israel Journal of Chemistry, 50(3):267-276, 2010.

[5] Juhan M Kahk, Neil V Rees, Jeseelan Pillay, Robert Tshikhudo, Sibulelo Vilakazi, and Richard G Compton. Electron transfer kinetics at single nanoparticles. Nano Today, 7(3):174-179, 2012. 
[6] Emma JE Stuart, Neil V Rees, and Richard G Compton. Particle-impact voltammetry: The reduction of hydrogen peroxide at silver nanoparticles impacting a carbon electrode. Chemical Physics Letters, 531:94-97, 2012.

[7] Hongjun Zhou, Jun Hui Park, Fu-Ren F Fan, and Allen J Bard. Observation of single metal nanoparticle collisions by open circuit (mixed) potential changes at an ultramicroelectrode. Journal of the American Chemical Society, 134(32):13212-13215, 2012.

[8] Yi-Ge Zhou, Neil V Rees, and Richard G Compton. The electrochemical detection and characterization of silver nanoparticles in aqueous solution. Angewandte Chemie International Edition, 50(18):4219-4221, 2011.

[9] Alexey Yakushenko, Dirk Mayer, Johan Buitenhuis, Andreas Offenhäusser, and Bernhard Wolfrum. Electrochemical artifacts originating from nanoparticle contamination by ag/agcl quasi-reference electrodes. Lab on a Chip, 2013.

[10] PA Bobbert, MM Wind, and J Vlieger. Diffusion to a slowly growing truncated sphere on a substrate. Physica A: Statistical Mechanics and its Applications, 141(1):58-72, 1987.

[11] Ian Streeter and Richard G Compton. Diffusion-limited currents to nanoparticles of various shapes supported on an electrode; spheres, hemispheres, and distorted spheres and hemispheres. The Journal of Physical Chemistry C, 111(49):18049-18054, 2007.

[12] Ian Streeter, Ronan Baron, and Richard G Compton. Voltammetry at nanoparticle and microparticle modified electrodes: theory and experiment. The Journal of Physical Chemistry C, 111(45):17008-17014, 2007.

[13] Stephen R Belding and Richard G Compton. Transient voltammetry at electrodes modified with a random array of spherical nanoparticles: Theory. The Journal of Physical Chemistry C, 114(18):8309-8319, 2010.

[14] Kristopher R Ward, Nathan S Lawrence, R Seth Hartshorne, and Richard G Compton. Modelling the steady state voltammetry of a single spherical nanoparticle on a surface. Journal of Electroanalytical Chemistry, 683:3742,2012 .

[15] Martin C Henstridge, Kristopher R Ward, and Richard G Compton. The marcus-hush model of electrode kinetics at a single nanoparticle. Journal of Electroanalytical Chemistry, 712:14-18, 2014.

[16] Walter Schottky. Über spontane stromschwankungen in verschiedenen elektrizitätsleitern. Annalen der Physik, 362(23):541-567, 1918.

[17] Ya M Blanter and Marcus Büttiker. Shot noise in mesoscopic conductors. Physics Reports, 336(1):1-166, 2000. 
[18] Enno Kätelhön, Kay J Krause, Pradyumna S Singh, Serge G Lemay, and Bernhard Wolfrum. Noise characteristics of nano-scaled redox-cycling sensors: Investigations based on random walks. Journal of the American Chemical Society, 135:8874-8881, 2013.

[19] William M. Haynes, editor. CRC Handbook of Chemistry and Physics, 93rd Edition. CRC Press, Taylor \& Francis Group, 2012. 


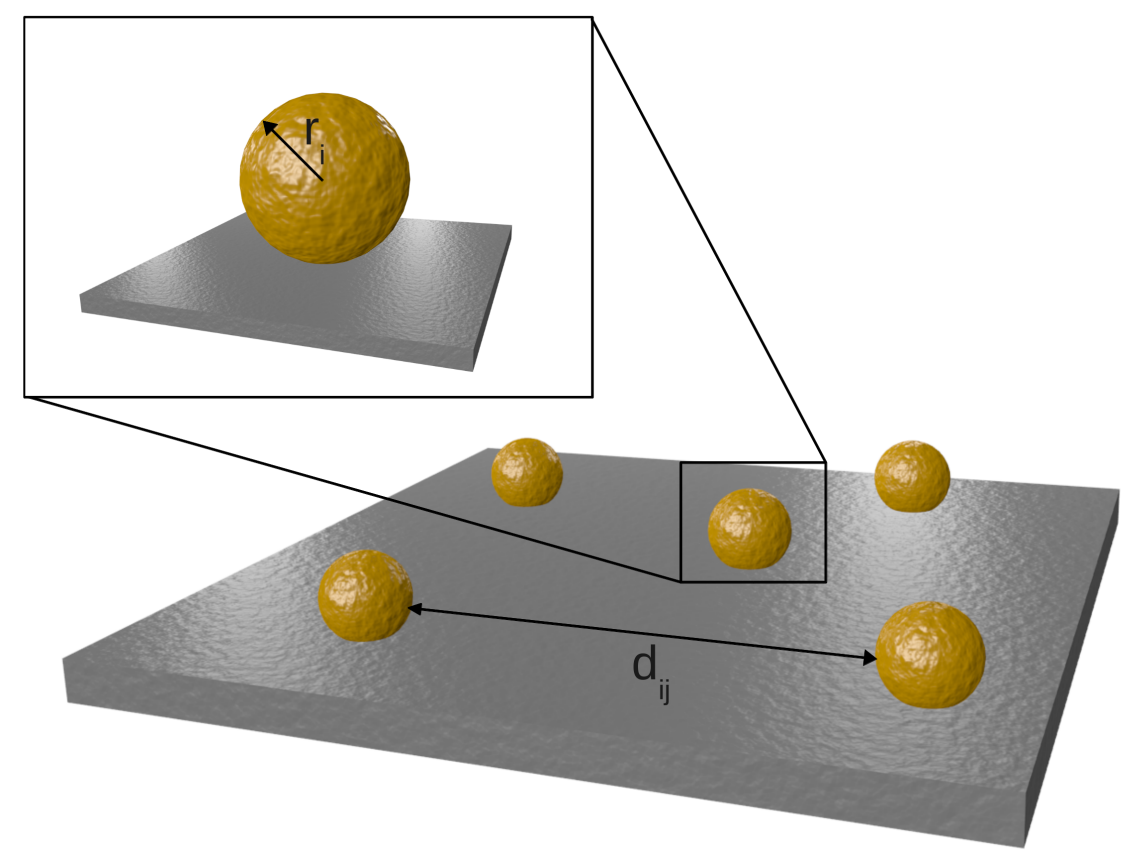

Figure 1: Schematic of the analysed geometry of spherical electrodes on a supporting surface. 


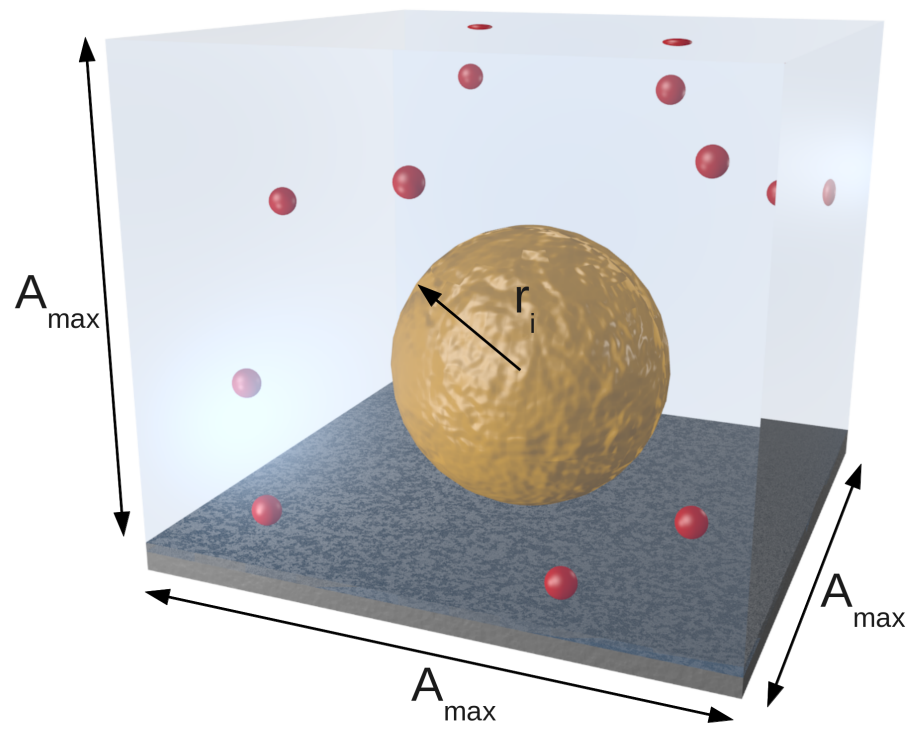

Figure 2: Schematic of the geometry that is simulated by random walks at the time $t=0$. Dimensions are not to scale. 


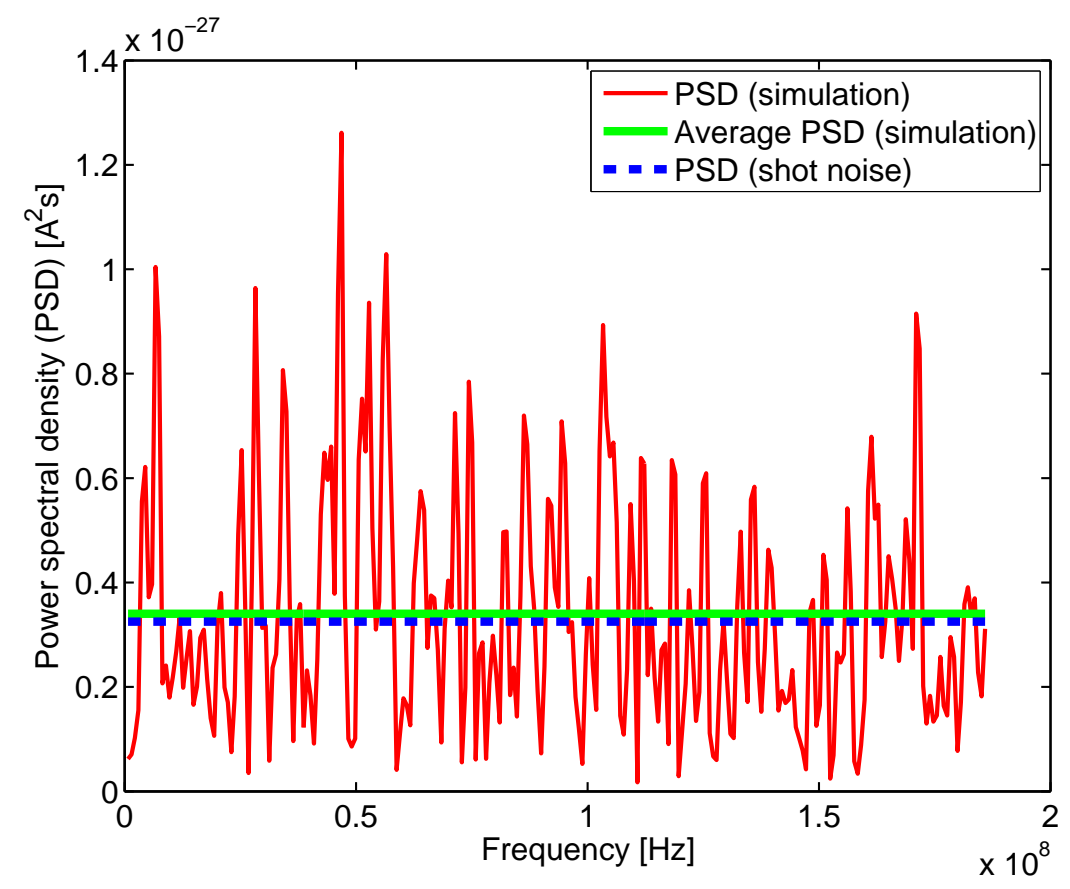

Figure 3: Comparison between the simulated- and the theoretically predicted power spectral density. The latter graph is calculated through Equation 5 using the average current $I_{\text {lim }}$ that is obtained in the respective simulation. The presented plot shows the power spectral density at a bulk analyte concentration of $6.2 \mathrm{mM}$. 


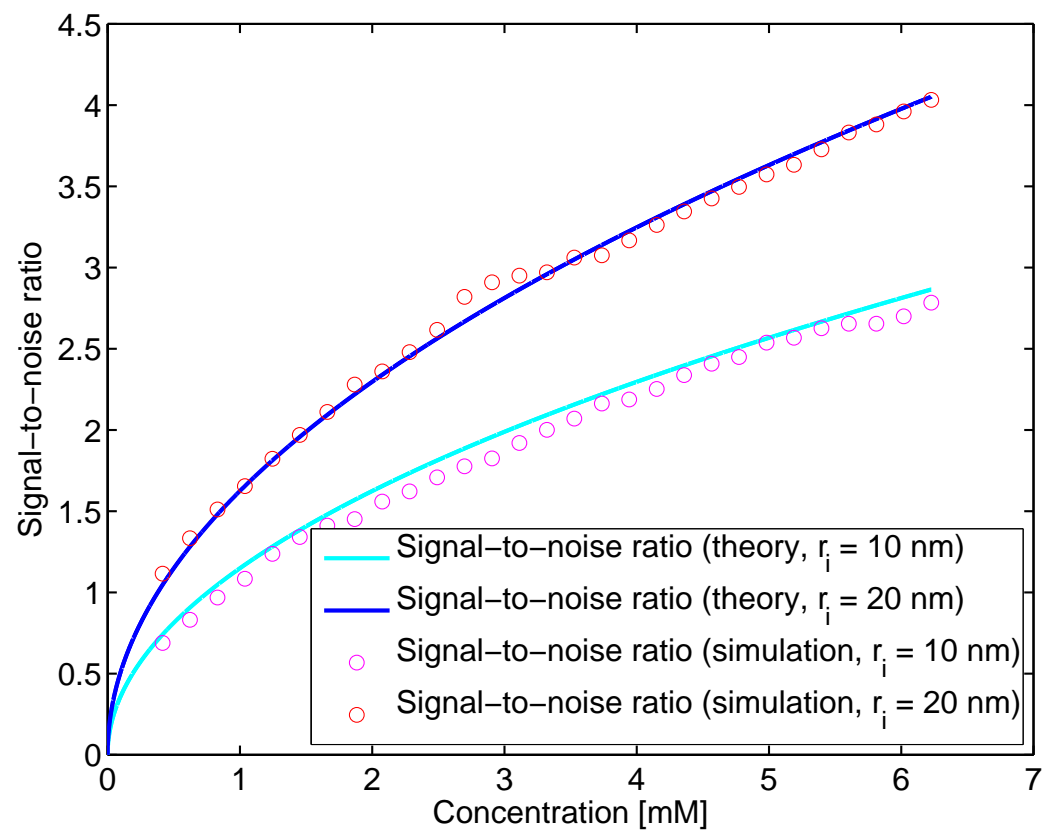

Figure 4: Comparison between the simulated and theoretically obtained signalto-noise ratios for two different particle radii $\mathrm{r}_{i}$ at varied analyte concentrations. The theoretical result is calculated through Equation 7.

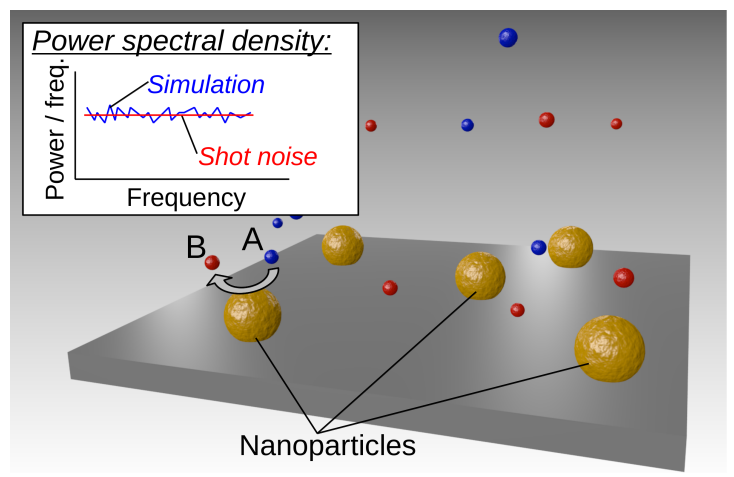

Figure 5: TOC figure. 